\title{
Image Fusion by Modified Spatial Frequency and Nonsubsampled Shearlet Transform
}

\author{
Jianhua Liu and Meng Gao \\ College of Electrical and Electronic Engineering, Shijiazhuang Tiedao University \\ ljhsjz@sohu.com
}

\begin{abstract}
It is now widely acknowledged that traditional wavelets are not very effective in dealing with multidimensional signals containing distributed discontinuities. Nonsubsampled Shearlet Transform is a new discrete multiscale directional representation, which combines the power of multiscale methods with a unique ability to capture the geometry of multidimensional data and is optimally efficient in representing images containing edges. In this work, coefficients with greater Modified-spatial frequency are selected to combine images when high-frequency and low-frequency Shearlet subbands of source images are compared. Experiments demonstrate that the method based on nonsubsampled shearlet transform and modified-spatial frequency is very competitive and better than other multi-scale geometric analysis tools in multifocus image fusion both in terms of objectives performance and objective criteria.
\end{abstract}

Keywords: Shearlet, MSF, Image fusion

\section{Introduction}

The purpose of image fusion is to combine information from multiple images of the same scene into a single image that ideally contains all the important features from each of original images. The resulting fused image will be thus more suitable for human and machine perception or for further image processing tasks. Nowadays, lots of research work on image processing is concentrated in the multiscale transform. Wavelet thresholding has been presented as a true signal estimation technique that exploits the capabilities of wavelet transform (DWT) for signal denoising [2-3]. The main advantage of traditional wavelet transform is its capability to capture different frequency components of an image, separately. Due to good spectral and spatial locality, wavelet transform can provide better image denoising performance. However, the DWT it is less effective in expressing the sharp transitions such as image edge and curve singularities due to its limitation of direction [4]. After this, a theory for multidimensional data called as MGA has been developed such as curvelet [5], bandelet [6], contourlet [7], nonsubsampled contourlet transform (NSCT) [8] etc. These new MGA tools provide higher directional sensitivity than wavelets. However, the curvelet, bandelet, contourlet lack of shift-invariance and results in artifacts along the edges to some extend. To represent the edges more efficiently, Labate et al., introduced a new multiscale analysis tool called shearlet that has all properties like other MGA tools as multiscale, localization, anisotropy and directionality [9]. Easley et al., [10] proposed nonsubsampled shearlet transform (NSST) that is realized by nonsubsampled laplacian pyramid (NSLP) and several shearing filters. The NSST also provides more flexible directional selectivity and shift in-variance. In this paper, a new multi-focus image fusion algorithm based on nonsubsampled shearlet and modified spatial frequency (MSF) [8] method was proposed. The NSST coefficients with greater MSF are used to fuse sources images. 


\section{Shearlet Transform}

The NSST is an extension of wavelet transform in multidimensional and multidirectional case which combines the multiscale and direction analysis, separately [9]. In NSCT, non-subsampled laplacian pyramid (NSLP) is used to decompose an image into low and high-frequency components, and then direction filtering is employed to get the different subbands and different direction shearlet coefficients. Direction filtering is achieved using the shear matrix which provides many more directions. In order to eliminate the effect of up-sampling and subsampling, NSST adopted NSLP filters as a substitute for the Laplacian pyramid filters used in the Shearlet transform [10], so that it has superior performance in shift-invariance, multi-scale and multi-directional properties. The discretization process of NSST is composed of two phases including multi-scale factorization and multi-directional factorization. NSLP is utilized to complete multi-scale factorization. The first phase ensures the multi-scale property by using two-channel nonsubsampled filter bank, and one low frequency image and one high frequency image can be produced at each NSLP decomposition level. Subsequently, NSLP are implemented to decompose the low frequency component available iteratively to capture the singularities in the image. The multi-directional factorization in NSST is realized via improved SF. These filters are formed by avoiding the sub-sampling to satisfy the property of shift-invariance. Figure 1, illustrates two level of NSST decomposition of an image. The number of shearing directions is set as 8,8 and 4 from finer scale to coarser scale.

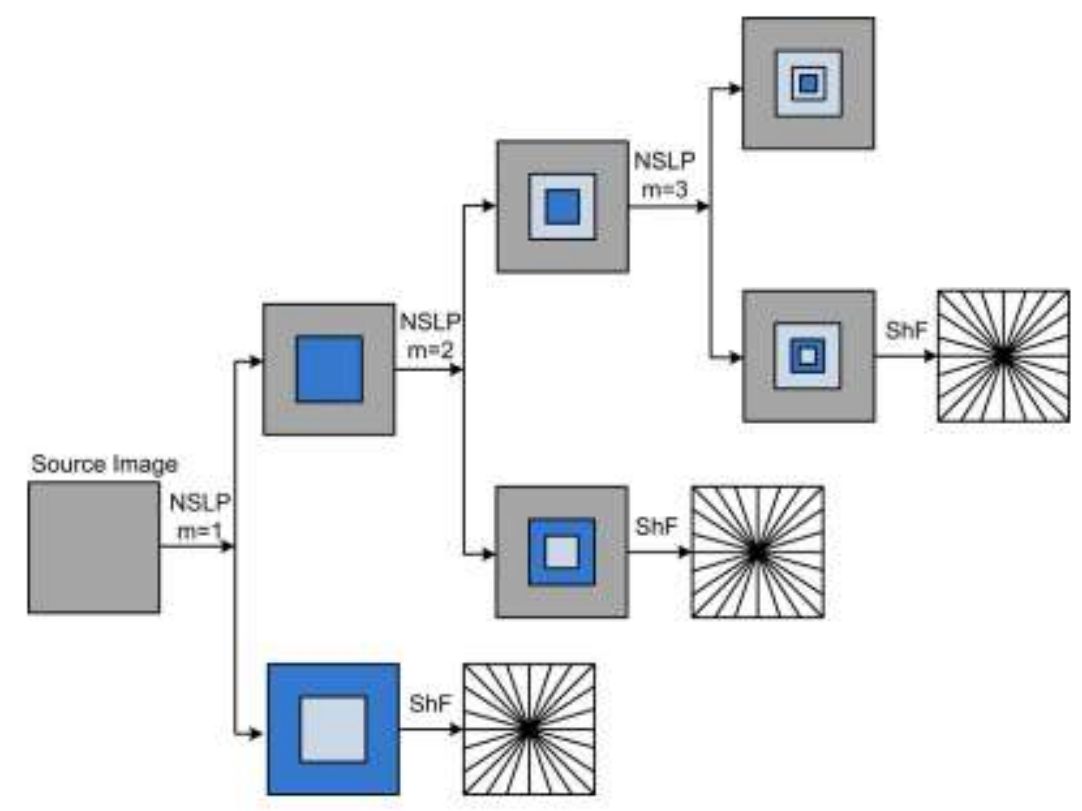

Figure 1. An Illustration of NSST Decomposition

\section{Modified Spatial Frequency}

Spatial frequency (SF) is calculated according to row and column frequency of the image. The image fusion method based on the SF has been proposed by Qu [8-11]. In this paper, the method of SF motivated the PCNN is effective to fuse the multifocus image. However, this method is not effective to fuse the multimodal medical images. The SF of the image is not enough to express the salience character because of lacks of direction information present in the image. The modified spatial frequency is capable of capturing fine details present in the image because of incorporate the direction information and the 
row and column frequency. The spatial frequency can be calculated according to the Equation (1).

$S F=\frac{1}{M N} \sum_{m=1}^{M} \sum_{n=2}^{N}\left(I_{m, n}-I_{m, n-1}\right)^{2}+\left(I_{m, n}-I_{m-1}\right)^{2}$

Where $I_{m, n}$ is the pixel value of the image in m column and $\mathrm{n}$ row in the image I with $\mathrm{M}$ column and $\mathrm{N}$ row. In this paper, the modified spatial frequency is adopted to evaluate the salience feature in the coefficient. The MSF consists of traditional spatial frequency (SF) and diagonal frequency (DF). The DF can be expressed as following.

$$
D F=\sqrt{\frac{1}{M N} \sum_{m=1}^{M} \sum_{n=1}^{N}\left(I_{m, n}-I_{m-, n-1}\right)^{2}+\left(I_{m-1, n}-I_{m, n-1}\right)^{2}}
$$

Then, modified spatial frequency can be calculated as:

$$
M S F=\sqrt{S F_{m, n}^{2}+D F_{m, n}^{2}}
$$

\section{Proposed Algorithm}

The present image fusion algorithm is demonstrated in Figure 2. The fusion steps can be generalized as following.

Step 1: The source images for fusion experiments are matched to each other.

Step 2: Source images images A and B are decomposed by the NSST, respectively, into one lowpass subband and highpass subband. Let $N S_{\mathrm{A}}^{l, k}(\mathrm{~m}, \mathrm{n})$ and $N S_{\mathrm{B}}^{l, k}(\mathrm{~m}, \mathrm{n})$ represent the coefficients of source images A and B at l-level and k-direction by NSST, respectively.

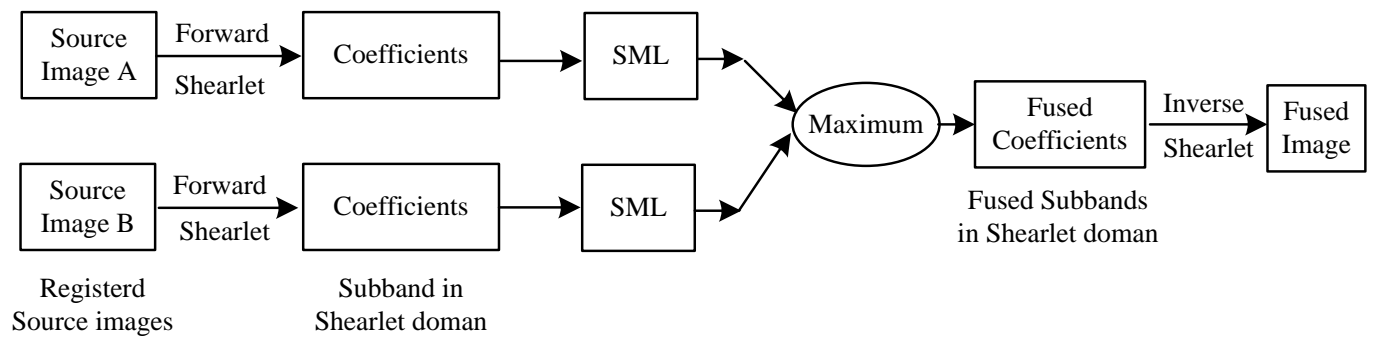

Figure 2. Schematic Diagram of NSST-MSF Fusion Algorithm

Step 3: Calculate MSFAl,k and MSFB1,k value of every NSST subband of the images.

Step 4: Then, the final fused coefficient will be decided by the Equation (3) and (4) which means the lager is the MSF, the NSST coefficient will be transported to final fused coefficient.

$$
\begin{aligned}
& \operatorname{Map}^{1, \mathrm{k}}(\mathrm{m}, \mathrm{n})=\left\{\begin{array}{l}
1 \operatorname{MSF}_{\mathrm{A}}^{1, \mathrm{k}}(\mathrm{m}, \mathrm{n}) \geq \operatorname{MSF}_{\mathrm{B}}^{1, \mathrm{k}}(\mathrm{m}, \mathrm{n}) \\
0 \operatorname{MSF}_{\mathrm{A}}^{1, \mathrm{k}}(\mathrm{m}, \mathrm{n})<\operatorname{MSF}_{\mathrm{B}}^{1, \mathrm{k}}(\mathrm{m}, \mathrm{n})
\end{array}\right. \\
& N S_{F}^{l, k}(\mathrm{~m}, \mathrm{n})=\left\{\begin{array}{l}
N S_{A}^{l, k}(\mathrm{~m}, \mathrm{n}) \text { if } \operatorname{Map}^{l, k}(\mathrm{~m}, \mathrm{n})=1 \\
N S_{B}^{l, k}(\mathrm{~m}, \mathrm{n}) \text { if } \operatorname{Map}^{l, k}(\mathrm{~m}, \mathrm{n})=0
\end{array}\right.
\end{aligned}
$$

Where $N S_{F}^{l, k}(\mathrm{~m}, \mathrm{n})$, and $N S_{\mathrm{B}}^{l, k}(\mathrm{~m}, \mathrm{n}) N S_{\mathrm{A}}^{l, k}(\mathrm{~m}, \mathrm{n})$ are the coefficients of the fused image $\mathrm{F}, \mathrm{A}$ and $\mathrm{B}$, respectively. 
Step 5: Reconstruct the fused image by adopting fused the NSST coefficients by inverse NSST.

\section{Experiments}

The two pairs of images showed in Figure 3, are used as source images to be fused in multi-focus images. In this paper, we compare this method of ShearletMSF with the CS-SFLCT-SML method proposed by Qu and NSCT method based on the MSF. In the direction of the filter decomposition, the decomposition parameter is set as $[2,3,4,4]$. The shift distance are set as $\mathrm{M}=\mathrm{N}=[-1,-2,-4,-8,1,2,4,8]$. The $\mathrm{M}$ is the shift distance in $\mathrm{x}$ axis and the $\mathrm{N}$ is the shift distance in $\mathrm{y}$ axis. In NSCT decomposition, $[2,3,4,4]$ is used as the vector of numbers of directional filter bank decomposition levels at each pyramidal level (from coarse to fine scale). The filter name for the directional decomposition step is 'pkva'. The name of the pyramid 2D filters is 'maxflat'. In the NSST, the 'pyr' filter is adopted in the NSLP. $[2,3,4,4]$ is used that indicates the $[4,8,16,16]$ directions are decomposed by the nonsubsampled shear filter. After NSST decomposition of the image A and B, the MSF rule is used to each subband. The experiment shows the MSF fusion rule in the every subband is better than the NSCT fusion method adopting the MSF rule.

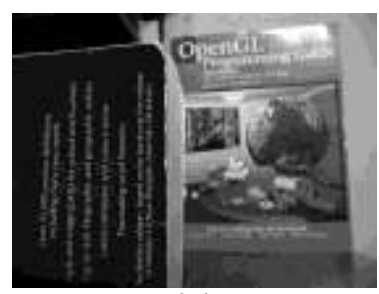

(a)

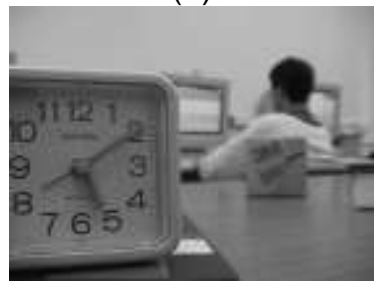

(e)

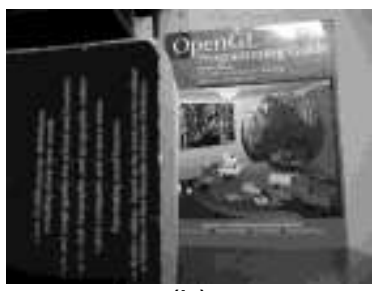

(b)

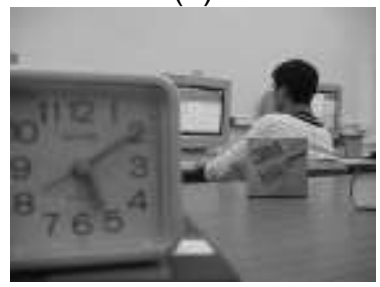

(f)

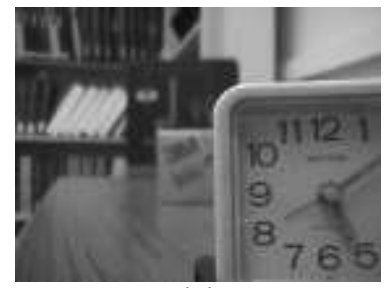

(c)

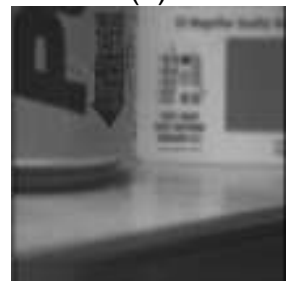

(g)

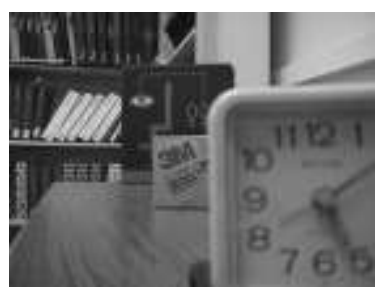

(d)

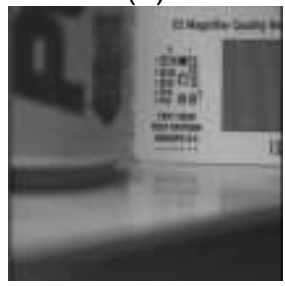

(h)

Figure 3. Source Images for Fused Experiments

Figure 4(a)-(c), and Figure 5(d)-(f), illustrates the fused images by proposed method and other two methods mentioned above. In order to clearly distinguish the distinction of the fusion results, the difference images between source image and the fused results fused by four algorithms are illustrated in the Figure 4(a)-(c), and Figure 5(d)-(f). If we subtract source image from the fused image, the residue image should be close to zero in well focused part. Hence, less residual in residue image mean more information in well focused part of the source images are almost fused into final image. Figure 4(f), and Figure 5(f), demonstrate the difference image of near zero in the relevant part. The difference image comparisons show the proposed scheme is most effective to fused the multi-focus images in the four algorithms. For further comparison except for the visual observation above, two objective metrics of the mutual information (MI) [12] and edge information $\mathrm{Q}^{\mathrm{AB} / \mathrm{F}}$ [13] are introduced to evaluate the four schemes. QAB/F measures how much edge information transferred from the source images to final merged images. MI evaluates the amount of information from source images is converted into the fusion result. The larger the $\mathrm{MI}$ and $\mathrm{Q}^{\mathrm{AB} / \mathrm{F}}$ value are, the better the fusion method is. The $\mathrm{MI}$ and $\mathrm{Q}^{\mathrm{AB} / \mathrm{F}}$ values of different fusion schemes are demonstrated in Table 1. It 
is obvious that the $\mathrm{MI}$ and $\mathrm{Q}^{\mathrm{AB} / \mathrm{F}}$ values of four fused images by the presented algorithms is larger than those of by the other three schemes. Generally speaking, just as Table 1, and Figure 4-Figure 5, we may objectively draw the conclusion that the proposed algorithm can preferably extract focus image part and discard the defocused region in source images according to both visual performance and objective criteria in the three methods.

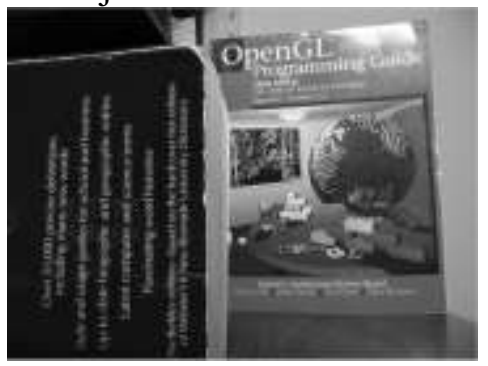

(a)CS-SFLCT-SML

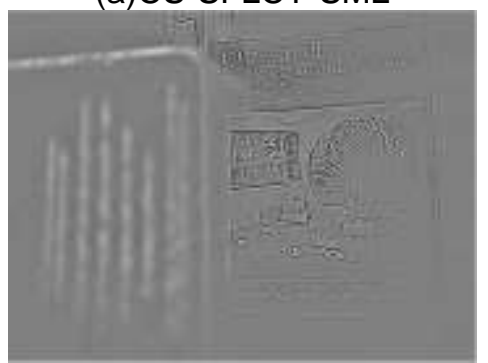

(d)CS-SFLCT-SML

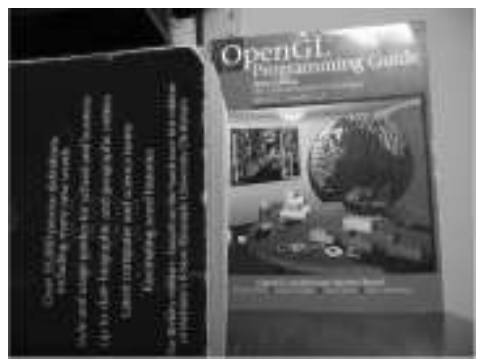

(b) NSCT-MSF

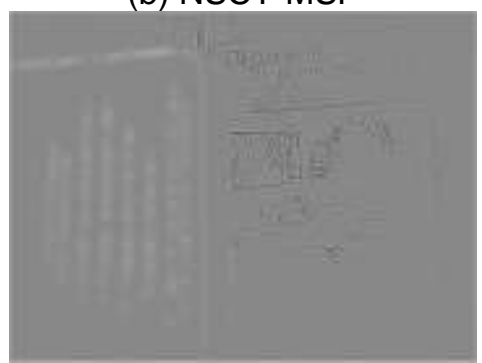

(e) NSCT-MSF

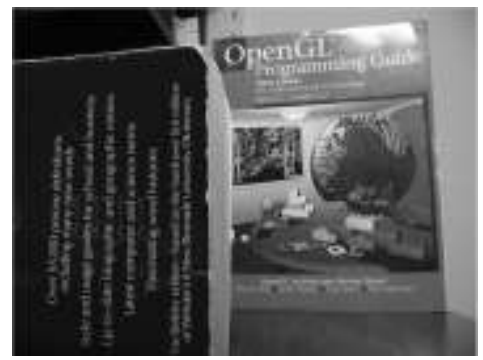

(c)NSST-MSF

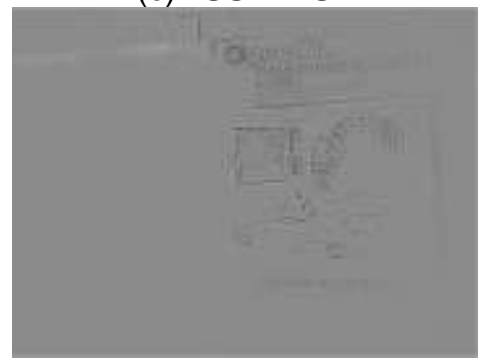

(f)NSST-MSF

\section{Figure 4. The Contrast of Fused 'Book' Image by Different Methods}

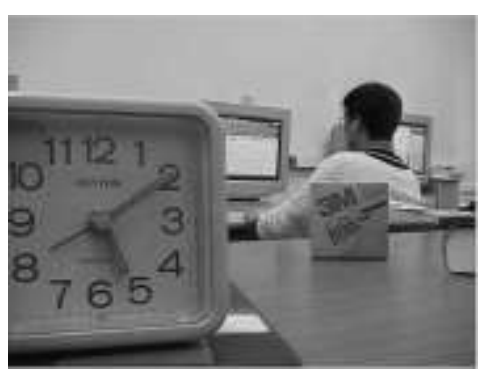

(a)CS-SFLCT-SML

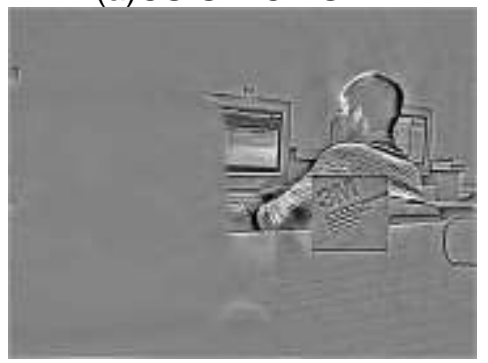

(d)CS-SFLCT-SML

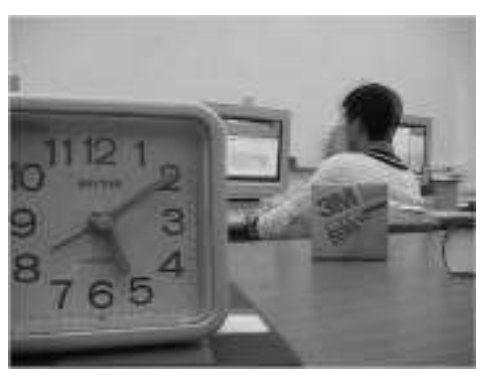

(b) NSCT-MSF

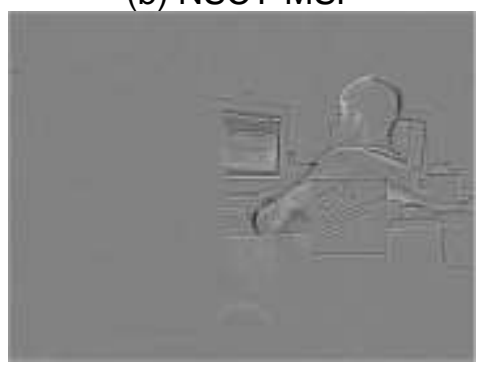

(e) NSCT-MSF

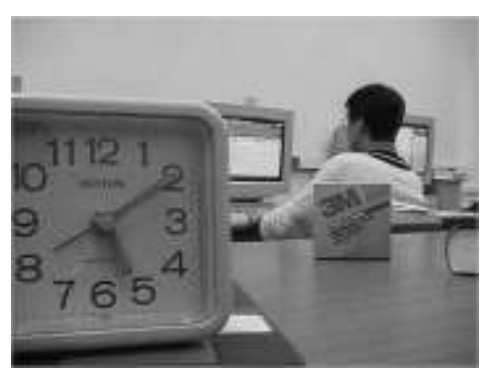

(c)NSST-MSF

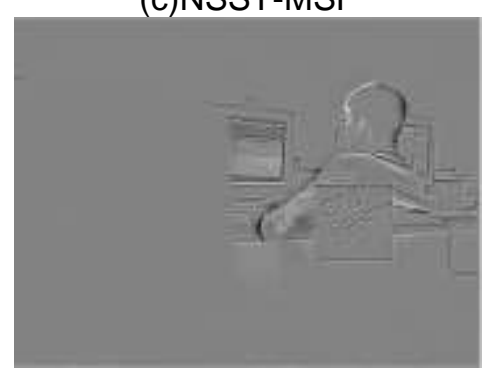

(f)NSST-MSF

Figure 5. The Contrast of Fused 'Lab' Image by Different Methods 
Table 1. Performance of Fusion by MSF in Different Transform Coefficients

\begin{tabular}{|c|c|c|c|c|}
\hline \multirow{2}{*}{ Images } & Criteria & $\begin{array}{c}\text { CS- } \\
\text { SFLCT+SML }\end{array}$ & NSCT+MSF & Shearlet+MSF \\
\hline \multirow{2}{*}{ Book } & MI & 7.0763 & 7.6521 & 7.8725 \\
\cline { 2 - 5 } & $\mathrm{Q}^{\mathrm{AB} / \mathrm{F}}$ & 0.7135 & 0.7391 & 0.7893 \\
\hline \multirow{2}{*}{ Lab } & $\mathrm{MI}$ & 6.8211 & 7.3592 & 7.5301 \\
\cline { 2 - 5 } & $\mathrm{Q}^{\mathrm{AB} / \mathrm{F}}$ & 0.7132 & 0.7901 & 0.8805 \\
\hline \multirow{2}{*}{ Pepsi } & $\mathrm{MI}$ & 6.6714 & 6.9403 & 7.2739 \\
\cline { 2 - 5 } & $\mathrm{Q}^{\mathrm{AB} / \mathrm{F}}$ & 0.7665 & 0.7708 & 0.7728 \\
\hline \multirow{2}{*}{ Disk } & $\mathrm{MI}$ & 6.1324 & 6.1544 & 6.9059 \\
\cline { 2 - 5 } & $\mathrm{Q}^{\mathrm{AB} / \mathrm{F}}$ & 0.6965 & 0.6915 & 0.7058 \\
\hline
\end{tabular}

\section{Conclusion}

In order to improve the effect of multifocus image fusion, a novel image fusion algorithm is presented in this paper. Nonsubsampled shearlet transform is adopted to decompose the source images to high pass coefficients and low pass coefficients because of nonsubsampled shearlet transform's ability to capture the geometry of multidimensional data and optimal efficiency in representing images containing edges. On the other side, the modified-spatial frequency is introduced into merging the NSST coefficient to final result image. The experiments on four pairs of multifocus demonstrate that the presented scheme is effective in merging the source multifocus image according to the subjective and objective performance valuation.

\section{References}

[1] S. Gabarda and G. Cristóbal, "On the use of a joint spatial-frequency representation for the fusion of multi-focus images", Pattern Recognition Letters, vol. 26, no. 16, (2005), pp. 2572-2578.

[2] Y, Chai, H. Li and Z. Li, "Multifocus image fusion scheme using focused region detection and multiresolution", Optics Communications, vol. 284, no. 19, (2011), pp. 4376-4389.

[3] D. D. Y Po and M. N. Do, "Directional multiscale modeling of images using the contourlet transform", IEEE Transactions on Image Processing, vol. 15, no. 6, (2006), pp. 1610-1620.

[4] K. Liu, L. Guo and J. Chen, "Image Fusion Algorithm Based on Contourlet Domain Hidden Markov Tree Models", Acta Photonica Sinica, vol. 39, no. 39, (2010), pp. 1383-1387.

[5] J. Zhou, A. L. Cunha and M. N. Do, "Nonsubsampled contourlet transform: Construction and application in enhancement", IEEE International Conference on Image Processing, (2005), pp. 469-472.

[6] Q. Zhang and B. L. Guo, "Multifocus image fusion using the nonsubsampled contourlet transform", Signal Processing, vol. 89, no. 7, (2009), pp. 1334-1346.

[7] G. Easley, D. Lim and W. Q. Lim, "Sparse directional image representations using the discrete Shearlet transform", Applied and Computational Harmonic Analysis, vol. 25, no. 1, (2008), pp. 25-46.

[8] X. Qu, J. Yan and G. Yang, "Sum-modified-Laplacian-based Multifocus Image Fusion Method in Sharp Frequency Localized Contourlet Transform Domain”, Optics and Precision Engineering, vol. 17, no. 5, (2009), pp. 1203-1202.

[9] P. Geng, Z. Wang, Z. Zhang and Z Xiao, "Image fusion by pulse couple neural network with shearlet mage fusion by pulse couple neural network with shearlet”, Optical Engineering, vol. 51, no. 6, (2015), pp. 067005-067005-7.

[10] G. R. Easley, L. Demetrio and C. Flavia, "Shearlet-based total variation diffusion for denoising", IEEE Transactions on Image Processing, vol. 18, no. 2, (2009), pp. 260-268..

[11] S. K. Nayar and Y. Nakagawa, "Shape from focus", IEEE Transactions on Pattern Analysis and Machine Intelligence, vol. 16, no. 8, (1994), pp. 824-831.

[12] G. Piella, "A general framework for multiresolution image fusion: from pixels to regions", Information Fusion, vol. 4, no. 4, (2004), pp. 259-280.

[13] C. S. Xydeas and V. Petrovic, "Objective image fusion performance measure", Electronics Letters, vol. 36, no. 4, (2000), pp. 308-309. 


\section{Authors}

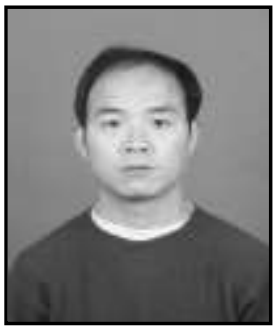

Jianhua Liu, he is a Ph.D. student of Donghua University in Shanghai. He is now an associate professor in College of Electrical and Electronic Engineering of Shijiazhuang Tiedao University in China. His research interests include computer application, include information acquisition and processing, and image processing. He has published more than 20 papers in journals and conferences. 
International Journal of Signal Processing, Image Processing and Pattern Recognition Vol. 10, No. 1 (2017) 